Pacific Journal of Mathematics

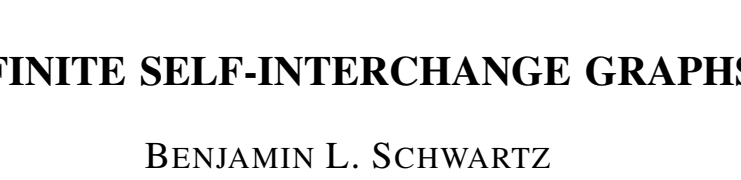




\section{INFINITE SELF-INTERCHANGE GRAPHS}

\section{B. L. SChwARTZ}

Let $G$ be an unoriented graph. Let $I(G)$ denote the interchange graph of $G$. If $G=I(G)$, we shall say $G$ is a selfinterchange graph (SIG). If for some positive integer $m \geqq 1$, we have $I^{m}(G)=G$, we shall say $G$ is eventually self interchange (ESIG). This paper extends previous results to characterize all finite degree SIG's and ESIG's, (loops and parallel edges permitted), finite or infinite, connected or disconnected. It will be seen that when infinite graphs are considered, several earlier results change. For example, there are ESIG's which are not SIG's; and loop-free SIG's which are not regular.

1. Terminology. In this paper, we shall use the unmodified term graph to mean locally finite $s$-graph with loops permitted. The case of parallel edges forbidden will be denoted restricted graph. An elementary chain will be called a line. The interchange operation is so defined that the interchange of a loop is again a loop ${ }^{1}$. A loop is considered a complete 1-graph ${ }^{1}$. A loop contributes 1 to the degree of its vertex ${ }^{1}$. If a graph $G$ has two parallel edges, the corresponding two vertices of $I(G)$ are likewise joined by two parallel edges ${ }^{1}$.

Definition. Let $G$ be a graph. Suppose the components of $G$ are $\left\{G_{i} \mid i \in A\right\}$ where $A$ is some index set. We shall say that $H$ is component-subgraph (hyphinated), or C-subgraph for short, if and only if components of $H$ are $\left\{G_{i} \mid i \in B\right\}$ where $B$ is some subset of $A$.

Similarly, if $G^{\prime}$ and $G^{\prime \prime}$ are disjoint graphs whose components are $\left\{G_{i} \mid i \in A\right\}$ and $\left\{G_{i} \mid i \in B\right\}$ respectively, where $A$ and $B$ are disjoint index sets, we say the graph consisting of the components of $G^{\prime}$ and the components of $G^{\prime \prime},\left\{G_{i} \mid i \in A \cup B\right\}$ is the $C$-union of $G^{\prime}$ and $G^{\prime \prime}$. Where context makes it clear, we shall sometimes write this using the ordinary union symbol $U$, e.g. $G^{\prime} \cup G^{\prime \prime}$.

2. Preliminaries. It has sometimes been asserted that $G$ is a SIG if and only if $G$ is regular of degree 2 [8], [12]. This assertion is valid only if the hypothesis include that $G$ is loop-free and finite. Nonregular SIG's with loops have been known for some time [5]. The author has elsewhere [17] characterized all finite connected $s$-graph SIG's (loops permitted). We restate the result here for later use: all

${ }^{1}$ For the present purpose, these conventions appear to be the most appropriate, although we recognize that other conventions for these concepts are sometimes used. 
finite, connected ESIG's are SIG's; and the only finite connected SIG's are graphs of the form of Figures (1c), $(2 b)$ or (2c) below.

The extension to infinite graphs is given in the following sections. The passage from finite to infinite graphs requires that certain existing tools be sharpened, since much of the current literature on interchange graphs applies only for finite ones, and sometimes to restricted and/or loop-free graphs [1], [7], [9], [11], [16].

The main result we use is an extension of a result of Krausz, originally given for finite restricted loop-free graphs [10]. Alternate approaches would involve extending various other theorems from the finite to the infinite case. One reviewer of this manuscript has suggested a result of van Rooij and Wilf [16] as one such possibility. While this would be quite possible, we believe our method is equally suitable.

The theorem of Krausz is stated for restricted finite loop-free graphs in [7] as follows:

Krausz' Theorem. A graph $H$ is an interchange graph of (another) graph $G$ if and only if there exists an edge-disjoint partition of the edges of $H$ into complete subgraphs, such that no vertex of $G$ lies in more than two of those subgraphs.

We now state and prove the following extension to locally finite $s$-graphs with loops.

Extended Krausz' Theorem. A locally finite s-graph $G$ is an interchange graph if and only if it has no vertex with two or more loops, and there is an edge-disjoint partition of its edges into a set of complete graphs such that no vertex of $G$ is in more than two of these subgraphs.

Proof: It is obviously sufficient to consider only connected graphs of degree $>1$.

The proof of Ore in [14] applies word for word to establish the following:

If $G$ is a graph, then $I(G)$ has an edge-disjoint partition into complete graphs in which no vertex is in more than two of these subgraphs. (It remains undetermined whether $I(G)$ can have multiple loops.) Conversely, if $H$ is a graph in which such an edge-disjoint partition exists, and no vertex of $H$ has two loops, then there is a graph $G$ such that $I(G)=H$.

The only thing remaining to prove is that an interchange graph cannot have any vertex with two or more loops. For this, we invoke the following obvious: 
Lemma. For any finite graph $G$, the number of loops in $I(G)$ is the same as the number of loops in $G$.

Now suppose $H=I(G)$ is an interchange graph with some vertex $\alpha$ incident to two loops. Since $H$ can be decomposed into complete graphs $K_{i}$ (by Ore's argument) and $\alpha$ can be included in only two of the $K_{i}$, it follows that no other edge of $H$ is incident to $\alpha$. Hence one component $H_{0}$ of $H$, must consist of just these two loops, Figure (2e). But by the lemma, the corresponding component $G_{0}$ of $G$ must have two loops, and therefore at least two edges. Hence by definition of the $I$ operation $I\left(G_{0}\right)=H_{0}$ has at least two vertices. But Figure (2e) has only one vertex. This contradiction completes the proof.

3. Graphs of degree 2. For reference, we catalog here certain graphs referred to later. These include all possible connected graphs of degree less than 3. In Figure 1 are the graphs of degree 0 , and

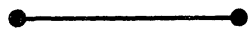

(b)

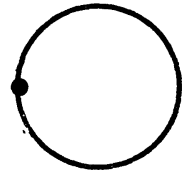

(c)

Figure 1.

1. The only graph of degree 0 is Figure (1a), the isolated vertex. The two graphs of degree 1 are in Figure (1b) and (1c): a line of length 1 and a loop, respectively. Figure 2 shows all the finite graphs of degree 2. These include a line of arbitrary length $\geqq 2$ (Figure (2a));

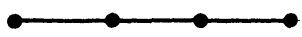

(a)

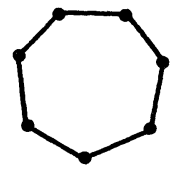

(b)

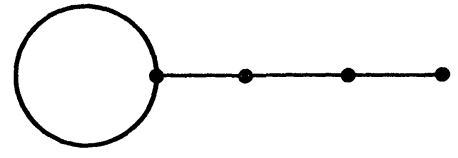

(c)

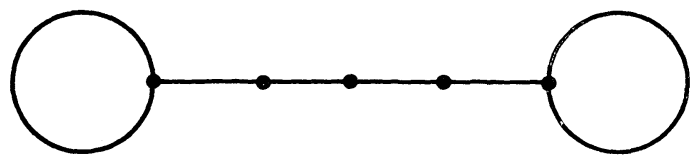

(d)

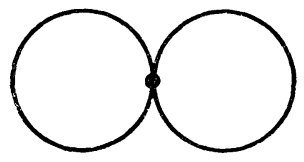

(e)

Figure 2. 
a cycle of arbitrary length $\geqq 2$ (Figure $(2 b)$ ); a line of arbitrary length $\geqq 1$ with a loop adjoined to one end (Figure (2c)); a line of arbitrary length $>1$ with a loop adjoined to each end (Figure (2d)); and two loops on the same vertex (Figure (2e)). Finally, Figure 3 shows the

(a)

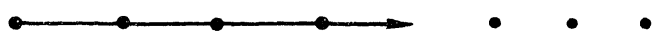

(b)

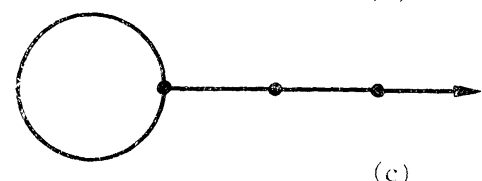

(c)

Figure 3.

infinite graphs of degree 2. These are: an infinite line whose edges can be consecutively numbered from $-\infty$ to $+\infty$ (Figure (3a)); a semiinfinite line, with edges numbered from 1 to $\infty$ (Figure (3b)); and a loop with a semi-infinite line adjoined (Figure (3c)).

4. Finite disconnected SIG's. Theorem 1. Let $G$ be a finite graph with $p$ distinct components $G_{1}, \cdots, G_{i}, \cdots, G_{p}$.

If $G$ is an ESIG, then $G_{i}$ is a SIG for $i=1, \cdots, p$. Hence, each $G_{i}$ is of either the form of Figure (1c), (2b), or (2c); and $G$ is a SIG.

Proof. The $I$ mapping clearly preserves connectivity. And since for a known fixed $m \geqq 1$, we have $I^{m}(G)=G$ each component $G_{i}$ is mapped into (another) component of $G$ by the function $I^{m}$, i.e., the mapping on the set of components $\left\{G_{i}\right\}$ induced by $I^{m}$ is a permutation of order $p$. From group theory, we know that this permutation is the product of disjoint (algebraic) cycles [3]. Let $r$ be the 1.c.m. of the orders of the algebraic cycles. Then $\left(I^{m}\right)^{r}=I^{m \cdot r}$ induces the identity mapping on the components $G_{i}$. Hence, for any component $G_{i}$, we have $I^{m r}\left(G_{i}\right)=G_{i}$; that is, $G_{i}$ is an ESIG.

But since $G_{i}$ is connected and finite, we can use the previous results cited in $\S 2$ to conclude that $G_{i}$ is a SIG, and of the form of Figure $(1 \mathrm{c}),(2 \mathrm{~b})$, or $(2 \mathrm{c})$.

5. Infinite SIG's first result. Now consider a general $G$. We require neither finiteness nor connectedness of $G$.

Theorem 2. Let $G$ be an ESIG. There exists a SIG, $H$ such 
that $G$ is a $C$-subgraph of $H$; and, if $G$ is of finite degree, so is $H$.

Proof. Let the components of $G$ be $\left\{G_{i} \mid i \in A\right\}$ for some index set $A$. The components of $H$ consist of the components of $G$, plus the components of $I^{j}(G)$ for $j=1,2, \cdots, m-1$ : viz

$$
H=\bigcup_{j=0}^{m-1}\left\{I^{j}(G)\right\} .
$$

The result is then immediate.

\section{Infinite SIG's of finite degree.}

Theorem 3. If $C$ is a SIG of finite degree, then $G$ is of degree $\leqq 2$. (This theorem is of interest only for infinite SIG's, since all finite SIG's have already been characterized in Theorem 1.)

Proof. If $G$ is a SIG of finite degree, clearly $I^{k}(G)$ is of the same degree for all $k$. Hence it suffices to prove that if degree $G>2$, then for some $k$, degree $I^{k}(G)$ is arbitrarily large. Menon [12] has proved that if $G$ is of degree $>3$, then $I^{k}(G)$ is of arbitrary high degree for sufficiently large $k$.

The case of degree 3 is more delicate.

Let $\alpha$ be a vertex of $G$ of degree 3. Let the three incident edges to $\alpha$ be denoted $a, b$, and $c$. By the extended Krausz' theorem, $\alpha$ is part of one or two complete graphs. Since there are three edges incident to $\alpha$, there are just two possible cases.

Case 1. All three edges are part of a complete graph. In this case, the complete graph clearly must be a complete 4-graph. Then $G$ must contain this complete 4-graph, $K_{4}$.

Case 2. Two edges, say $a$ and $b$, are part of a complete graph; and one edge, say $c$, is part of another complete graph. Then $a$ and $b$ are two edges of a complete 3-graph. And either $c$ is a loop, and

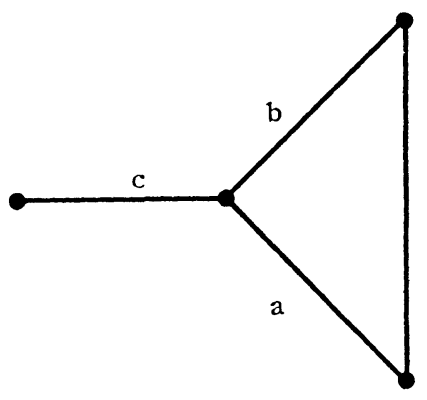

(a)

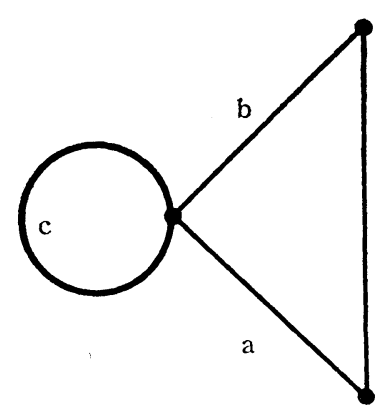

(b)

Figure 4. 
thus a complete 1-graph, or $c$ is a complete 2-graph of one edge. These two possible cases are illustrated in Figure 4.

By direct calculation, in each case, it is verified that $I\left(K_{4}\right), I^{2}(4 a)$, and $I^{2}(4 \mathrm{~b})$ all contain vertices of degree $\geqq 4$. By Menon's result, we have a contradiction. This completes the proof.

CoRollary. If $G$ is an ESIG of finite degree, then $G$ is of degree $\leqq 2$.

Proof. By Theorem 2, $G$ is a $C$-subgraph of a SIG, which fulfills the hypotheses of Theorem 3 .

\section{Connected infinite SIG's.}

THEOREM 4. Let $G$ be a connected infinite ESIG of finite degree. Then $G$ is of one of the three forms of Figure 3, and therefore is $a$ SIG.

Proof. By the corollary to Theorem 3, $G$ is of degree $\leqq 2$. Hence, the only candidates are the connected infinite graphs of degree 2; these are completely listed in Figure 3. By direct calculation, each of these is shown to be a SIG.

8. Disconnected infinite SIG's. We have shown that for connected graphs of finite degree, all ESIG's are SIG's. If the connectivity condition is dropped, the result no longer holds. However, in view of Theorem 2, it suffices to consider SIG's in the infinite disconnected case, since all ESIG's are $C$-subgraphs.

We shall use the notation $L_{i}$ to denote a line of length $i ; i=0$, $1,2, \cdots$. (Hence $L_{0}$ and $L_{1}$ are graphs of the forms Figure (1a) and (1b), respectively.) We shall denote graphs of the forms Figure (2d) and (2e) by $M_{i}$, where $i$ is the length of the line between the two loops.

The graph $\mathscr{L}=\bigcup_{i=0}^{\infty} L_{i}$ is obviously a SIG, as is immediately verified by direct calculation. We now show that this is essentially the only SIG of finite degree beyond those we have already described.

Lemma 1. For $i \geqq 0, L_{i}$ is a component of $I(G)$ if and only if $L_{i+1}$ is a component of $G[18]$.

Lemma 2. For $i>0, M_{i}$ is a component of $I(G)$ if and only if $M_{i-1}$ is a component of $G$.

The proofs of these lemmata are easy, and are omitted. 
Theorem 5. Let $G$ be a SIG of finite degree which does not contain as a $C$-subgraph any of the following forms:

Figure (2b), a cycle;

Figure (2c), a finite line with attached loop;

Figure (3a), an infinite line;

Figure (3b), a semi-infinite line;

Figure (3c), a semi-infinite line with attached loop; or

$$
\mathscr{L}=\bigcup_{i=0}^{\infty} L_{i}
$$

Then $G$ is the empty graph.

Proof. If $L_{i}, i>0$, is a component of $G$, then $\mathscr{Z}$ is a $C$-subgraph of $G$. This follows by infinite induction, using Lemma 1.

Since by hypothesis $G$ does not contain $\mathscr{P}$, we conclude that $G$ does not contain any $L_{i}, i=0,1,2, \cdots$, as a component.

All other components of degree less than 3 have been explicitly excluded except $M_{i}$.

If for some $i>0, M_{i}$ is a component of $G$, then $M_{i-1}$ is a component. This follows as above from Lemma 2.

Hence by finite induction, if $M_{i}$ is a component of $G$, so is $M_{0}$, (Figure (2e)). By the extended Krausz theorem, however, $M_{0}$ is not an interchange graph. But this is a contradiction.

It follows that $G$ contains no component $M_{i}$. Hence $G$ contains no component at all, and hence is empty. This completes the proof.

Corollary. A graph $G$ of finite degree is a SIG if and only if it is C-union of graphs of the form of (1c), (2b), (3a), (3b), (3c), and $\mathscr{L}$.

9. ESIG's that are not SIG's. From Theorem 5, we can characterize all the ESIG's of finite degree which are not SIG's. They are all $C$-subgraphs of the graph $\mathscr{L}$ of the previous section. Let $G$ be an ESIG. Clearly if $L_{i}$ is a component of $G$, so is $I^{m}\left(L_{i}\right)=L_{i-m}$ for $m \leqq i$. It is therefore easy to see that any graph of the following form is an ESIG of this type.

$J_{m, n}=\bigcup_{i=0}^{\infty} L_{m i+n}$ where $m, n$ are positive integers and $n<m$. Furthermore, we have:

THEOREM 6. C-unions of these graphs $J_{m, n}$ are the only ESIG's of finite degree which do not contain any SIG $C$-subgraphs.

The proof follows the lines of the proof of Theorem 5. We omit the details. 
CoRollary. A graph $G$ of finite degree is an ESIG if and only if it is the C-union of graphs of the form of (1c), (2b), (2c), (3a), (3b), (3c), $\mathscr{L}$, and $J_{m, n}$.

The corollaries of this and the previous section provide the characterization of finite degree SIG's and ESIG's promised in the introduction.

10. Acknowledgement. The author gratefully acknowledges the contribution of the referee, who pointed out the necessity for distinguishing between the concepts of subgraph and $C$-subgraph. This has facilitated an improvement in both the clarity and the rigor of the presentation.

\section{BIBLIOGRAPHY}

1. A. Andreatta, On finite graphs which are line graphs (Italian), 1st Lombardo Accad. Sci. Lett. Remd. A. 98 (1964), 133-156.

2. Claude Berge, The theory of graphs and its applications, translated by Alison Doig, John Wiley and Son, 1962.

3. Garrett Birkhoff and Saunders MacLane, A Survey of modern algebra, revised ed., Macmillan, 1953.

4. G. Chartrand, The existence of complete cycles in repeated line graphs, Bull. Amer. Math. Soc. 71 (1965), 668-670.

5. Capt. Bernard Clark, USMC; private communication with the author, 1964.

6. Anna Maria Ghirlanda, Osservazioni sulle caratteristiche dei grafi o singrammi, Ann. Univ. Ferrara Sez. (N.S.) 11 (1962-65), 93-106.

7. Frank Harary, "Combinatorial problems in graphical enumeration", Chapter 6 of Applied combinatorial mathematics, Edwin. F. Beckenbach, editor, John Wiley and Sons, 1964.

8. Review of No. 16 below, Math. Review 33, No. 3959.

9. A. Hoffman and D. Ray-Chaudhuri, On the line graph of a finite affine plane, Canad. J. Math. 17 (1965), 687-694.

10. J. Krausz, Domenstration nouvelle d'une theoreme de Whitney sur les reseaux, Mat. Fiz. Lapok 50 (1943), 75-85.

11. V. Menon, The isomorphism between graphs and their adjoint graphs, Canad. Math. Bull 8 (1965), 7-15.

12. On repeated interchange graphs, Amer. Math. Monthly 13 (1966), 986-989.

13. L. Muracchini and A. Ghirlanda, Sul grafo commutato e sul grafo opposto di un grafo orientato, Atti. Sem. Mat. Fis. Univ. Modena 14 (1965), 87-97.

14. Oystein Ore, Theory of graphs, American Mathematical Society Colloquium Publications, Volume 38, Providence, R.I., 1962.

15. D. Ray-Chaudhuri, Characterization of line graphs, Math. Res. Ctr. Univ. Wis. Tech. Report 642, 1966.

16. A. van Rooij and H. Wilf, The interchange graph of a finite graph, Acta. Math. Acad. Sci. Hungar 16 (1965), 263-269.

17. B. L. Schwartz, On interchange graphs, Pacific J. Math. 27 (1968), 393-396.

18. H. Whitney Congruent graphs and the connectivity of graphs, Amer. J. Math. 54 (1932), 150-168.

Received September 23, 1968, and in revised form May 6, 1969. 


\section{PACIFIC JOURNAL OF MATHEMATICS}

\section{EDITORS}

\author{
H. ROYDEN \\ Stanford University \\ Stanford, California \\ Richard Pierce \\ University of Washington \\ Seattle, Washington 98105
}

\author{
J. DugundJI \\ Department of Mathematics \\ University of Southern California \\ Los Angeles, California 90007 \\ BASIL GoRDON \\ University of California \\ Los Angeles, California 90024
}

\section{ASSOCIATE EDITORS}
E. F. BECKENBACH
B. H. Neumann
F. WOLF
K. YoSHIDA

\section{SUPPORTING INSTITUTIONS}

\author{
UNIVERSITY OF BRITISH COLUMBIA \\ CALIFORNIA INSTITUTE OF TECHNOLOGY \\ UNIVERSITY OF CALIFORNIA \\ MONTANA STATE UNIVERSITY \\ UNIVERSITY OF NEVADA \\ NEW MEXICO STATE UNIVERSITY \\ OREGON STATE UNIVERSITY \\ UNIVERSITY OF OREGON \\ OSAKA UNIVERSITY \\ UNIVERSITY OF SOUTHERN CALIFORNIA
}

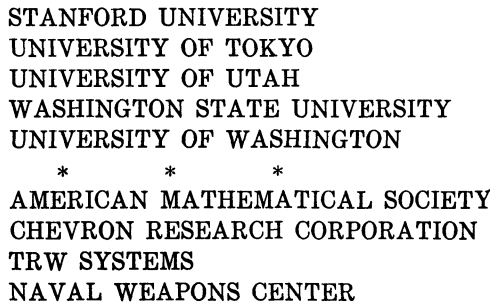

The Supporting Institutions listed above contribute to the cost of publication of this Journal, but they are not owners or publishers and have no responsibility for its content or policies.

Mathematical papers intended for publication in the Pacific Journal of Mathematics should be in typed form or offset-reproduced, double spaced with large margins. Underline Greek letters in red, German in green, and script in blue. The first paragraph or two must be capable of being used separately as a synopsis of the entire paper. It should not contain references to the bibliography. Manuscripts, in duplicate if possible, may be sent to any one of the four editors. Please classify according to the scheme of Math. Rev. 36, 1539-1546. All other communications to the editors should be addressed to the managing editor, Richard Arens, University of California, Los Angeles, California, 90024.

50 reprints are provided free for each article; additional copies may be obtained at cost in multiples of 50 .

The Pacific Journal of Mathematics is published monthly. Effective with Volume 16 the price per volume (3 numbers) is $\$ 8.00$; single issues, $\$ 3.00$. Special price for current issues to individual faculty members of supporting institutions and to individual members of the American Mathematical Society: $\$ 4.00$ per volume; single issues $\$ 1.50$. Back numbers are available.

Subscriptions, orders for back numbers, and changes of address should be sent to Pacific Journal of Mathematics, 103 Highland Boulevard, Berkeley, California, 94708.

PUBLISHED BY PACIFIC JOURNAL OF MATHEMATICS, A NON-PROFIT CORPORATION

Printed at Kokusai Bunken Insatsusha (International Academic Printing Co., Ltd.), 7-17, Fujimi 2-chome, Chiyoda-ku, Tokyo, Japan. 


\section{Pacific Journal of Mathematics}

\section{Vol. 31, No. $2 \quad$ December, 1969}

Efraim Pacillas Armendariz, Quasi-injective modules and stable torsion

classes..........................................

J. Adrian (John) Bondy, On Ulam's conjecture for separable graphs...

Vasily Cateforis and Francis Louis Sandomierski, On commutative rings over which the singular submodule is a direct summand for every module .....

Rafael Van Severen Chacon, Approximation of transformations with continuous

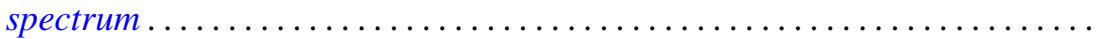

Raymond Frank Dickman and Alan Zame, Functionally compact spaces ...... 303

Ronald George Douglas and Walter Rudin, Approximation by inner

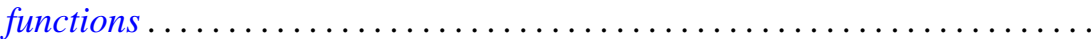

John Walter Duke, A note on the similarity of matrix and its conjugate

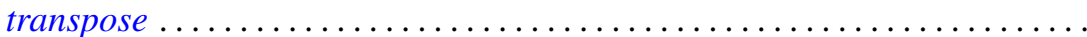

Micheal Neal Dyer and Allan John Sieradski, Coverings of mapping

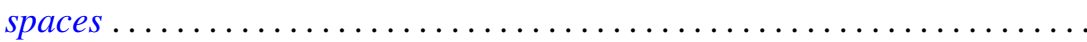

Donald Campbell Dykes, Weakly hypercentral subgroups of finite groups .....

Nancy Dykes, Mappings and realcompact spaces.....................

Edmund H. Feller and Richard Laham Gantos, Completely injective

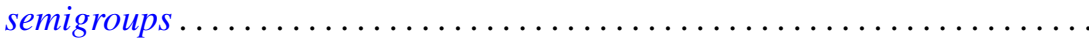

Irving Leonard Glicksberg, Semi-square-summable Fourier-Stieltjes

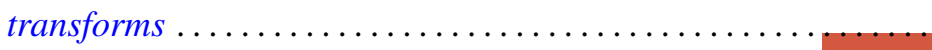

Samuel Irving Goldberg and Kentaro Yano, Integrability of almost cosymplectic structures...

Seymour Haber and Charles Freeman Osgood, On the sum $\sum\langle n \alpha\rangle^{-t}$ and numerical integration ..........................

Sav Roman Harasymiv, Dilations of rapidly decreasing functions ....

William Leonard Harkness and R. Shantaram, Convergence of a sequence of

transformations of distribution functions

Herbert Frederick Kreimer, Jr., A note on the outer Galois theory of rings ...

James Donald Kuelbs, Abstract Wiener spaces and applications to analysis. .

Roland Edwin Larson, Minimal $T_{0}$-spaces and minimal $T_{D}$-spaces...

A. Meir and Ambikeshwar Sharma, On Ilyeff's conjecture .

Isaac Namioka and Robert Ralph Phelps, Tensor products of compact convex sets....

James L. Rovnyak, On the theory of unbounded Toeplitz operators ....

Benjamin L. Schwartz, Infinite self-interchange graphs.......

George Szeto, On the Brauer splitting theorem...

Takayuki Tamura, Semigroups satisfying identity $x y=f(x$,

Kenneth Tolo, Factorizable semigroups .................. 